\title{
IMMUNOGENICITY AND SAFETY AFTER SARS-COV-2 VACCINE IN PRIMARY ANTIPHOSPHOLIPID SYNDROME (PAPS)
}

\begin{abstract}
Flavio Victor Signorelli1,2, , Gustavo Guimarães Moreira Balbi ${ }^{2,3}$, Vitor Antônio de Angeli Oliveira², Ana Luisa Cerqueira de Sant'Ana Costa $^{2}$, Ana Paula Rossi Gandara², Carolina Torres Ribeiro², Léonard de Vinci Kanda Kupa², Ana Cristina de Medeiros-Ribeiro², Nadia Emi Aikawa², Carla Gonçalves Schahin Saad², Emily Figueiredo Neves Yuki², Sandra Gofinet Pasoto², Clovis Artur Silva², Danieli Andrade², Eloisa Bonfáa
\end{abstract}

1.Universidade do Estado do Rio de Janeiro, Rio de Janeiro (RJ), Brazil.; 2.Universidade de São Paulo, São Paulo (SP), Brazil; 3.Universidade Federal de Juiz de Fora, Juiz de Fora (MG), Brazil.

${ }^{\star}$ Corresponding author: flasigno@hotmail.com

\section{BACKGROUND}

Immunogenicity data of COVID-19 vaccines for primary antiphospholipid syndrome (PAPS) is lacking. Recent reports of thrombosis with adenovirus-based vaccines raise concern in this predisposed population. Our aim was to evaluate the efficacy and safety of CoronaVac in PAPS patients.

\section{METHODS}

In this phase 4 prospective controlled trial, thrombotic PAPS patients were balanced by age $(p=0.982)$ and sex $(p=1.000)$ to a control group (CTRL) without autoimmune rheumatic disease or under immunosuppressive therapy. Participants positive to COVID-19 serology and/or neutralizing antibody ( $\mathrm{Nab}$ ) at baseline or those who tested positive for RT-PCR COVID-19 were excluded. Patients received a two-dose vaccine (D0 and D28) and blood samples were obtained at D0, D28 and D69 (6 weeks after second dose). Immunogenicity was evaluated by SARS-CoV-2 IgG seroconversion (SC), geometric mean titer (GMT), factor increaseGMT (FI-GMT), presence of $\mathrm{Nab}$ and its neutralizing activity (Nab-activity) at all time-points. Adverse events and incident cases of COVID-19 were registered.

\section{RESULTS}

Forty-four PAPS and 132 controls received the vaccine first dose. They were mostly women (86.4\%), median age of 46 years. Thirty-one PAPS and 108 controls were included in the immunogenicity analysis. At D28, a low and analogous seroconversion was observed for PAPS and CTRL (25.8\% vs. 30.6\%, $p=0.609$ ). A significant increase was observed at D69 with comparable SC (83.9\% vs. 93.5\%, $p=0.092)$, GMT $(50.2[95 \% \mathrm{Cl}=34.5-73.2]$ vs. $61.7[95 \% \mathrm{Cl}=52.8-72.3], \mathrm{p}=0.249)$ and FI-GMT (21.4 $[95 \% \mathrm{Cl}=14.5-31.6]$ vs. $26.5[95 \% \mathrm{Cl}=22.3-31.4], \mathrm{p}=0.586)$ in both groups. The frequency of $\mathrm{Nab}$ at D28 was lower in PAPS than CTRL $(16.1 \%$ vs. $35.2 \%, p<0.05)$, with a robust rise and comparable rates at D69 $(77.4 \%$ vs. $78.7 \%, p=0.440)$. Nab-activity was similar between PAPS patients vs. CTRL (38.1\% [32.0-55.5] vs. 43.7\% [34.2-66.4], $p=0.275)$ at D28 and (64.3 [49.0-77.0\%] vs. 60.9 [45.6-81.3\%], $p=0.689$ ) at D69. Four patients (1 PAPS and 3 CTRL) became mildly symptomatic and tested positive for RT-PCR COVID-19, until 4 days after second dose. Regarding safety, no moderate/severe adverse events occurred.

\section{CONCLUSION}

We demonstrated for the first time that CoronaVac two-dose vaccine elicits a very good antibody response with rates comparable to CTRL group. The excellent safety profile reinforces its recommendation for all PAPS patients.

\section{KEYWORDS}

Antiphospholipid syndrome, SARS-CoV-2, CoronaVac, COVID-19. 